\title{
Uncovering the Evolutionary Sequences for the C-J Stars Based on their Chemical Abundances
}

\author{
Ana Beatriz de Mello ${ }^{1}$ and Silvia Lorenz-Martins ${ }^{2}$ \\ ${ }^{1}$ Observatório Nacional - MCT, Brazil (email: demello@on.br), \\ ${ }^{2}$ Observatório do Valongo - UFRJ, Brazil
}

\begin{abstract}
We present chemical abundances obtained by fitting synthetic spectra to the FEROS data of $12 \mathrm{C}-\mathrm{J}$ stars, normal and silicate carbon one. The Li and ${ }^{13} \mathrm{C}$ abundance, as well as the sprocess elements abundance indicates no evidence of a diferent mechanism of formation between the two kinds of C-J stars. We also studied the $\alpha$ elements, and that suggests a scenario that put the carbon C-R stars as a possible progenitor of the beither C-J stars. We made also available a study of the radial velocity of some stars of the sample calculated at different epochs. That allowed us to make some discussion about the probable binary system of some silicon C-J carbon stars. Based on these abundances and radial velocities, we discuss possible evolutive sequences for the C-J.
\end{abstract}

Keywords. techniques: radial velocities, stars: abundances, stars: AGB and post-AGB, stars: carbon

\section{Introduction}

There are still many open questions about C-J type carbon stars. Estimates of sprocess elements abundances for these low ${ }^{12} \mathrm{C} /{ }^{13} \mathrm{C}$ stars suggest that they achieved their high carbon abundances through a mechanism other than the dredge-up processes that cause the carbon enrichment in the more common C-N carbon stars. C-J stars are also notable, among their chemical peculiarities, for abnormally high abundances of ${ }^{7} \mathrm{Li}$. Additionally, the circumstellar envelope of most C-J stars is carbon-rich, in accordance with their photospheric composition. However, there is a peculiar C-J stars referred as silicate carbon stars, due to an emission feature at $9,8 \mu \mathrm{m}$, typical of oxygen-rich stars. Because of these unique traits of the C-J class, little is known about how these objects evolve.

\section{Radial Velocity}

The radial velocity measures were applied to both correct the FEROS spectra for the chemical abundance analysis as well as to investigate the possibility of a binary nature to the C-J silicate carbon stars. Until now, the most plausible scenery for C-J silicate carbon stars seems to be a binary system, composed by an unseen, low-luminosity companion. The oxygen- rich material that was shed by mass loss when the primary star was an M giant can be stored in a circumbinary disk (Morris 1987, Evans 1990), or in a circumstellar disk around the low-luminosity companion (circum-companion disk, Yamamura et al. 2000) even after the primary star becomes a carbon star.

V433 Pup seems to have a certain variation in the radial velocity, however, small in comparison with the errors. MT Hya, MC79 2-11 and $\mathrm{C}^{*} 1003$ have a variation of $3.4 \mathrm{~km} \cdot \mathrm{s}^{-1}$ in 7 years, $3 \mathrm{~km} \cdot \mathrm{s}^{-1}$ in 3 years and $9,2 \mathrm{~km} \cdot \mathrm{s}^{-1}$ in 35 months, respectively, 
which points to a binary system that may enclose an oxigen-rich disk. BM Gem is one of the most studied star of our sample. All others either do not present any relevant variation at the radial velocity or do not have more the one measure.

\section{Chemical Abundances}

From all the element abundances calculated, the most relevant ones were the ${ }^{7} \mathrm{Li}$ and ${ }^{13} \mathrm{C}$, which are produced in the stellar interior and it only reach the outer part of the atmosphere where they are detected, if besides the dredge-up process also happens an extra mechanism of mixture. It is supposed that both abundances vary together (Abia et al. 1993).

The ${ }^{12} \mathrm{C} /{ }^{13} \mathrm{C}$ distribution of of the sample fluctuate around 5.5; and an average value for the C-J is 5.9; which is a close result to $4.7 \pm 2.8$ obtained by Ohnaka \& Tsuji (1999). Most of our targets has an uncommon abundance of ${ }^{7} \mathrm{Li}$ together with low ratios of ${ }^{12} \mathrm{C} /{ }^{13} \mathrm{C}$. Figure 1 presents the correlation of those parameters for our targets with the ones published by Abia \& Isern (2000). It is noticeable that our results do not flee from the behavior found by previous authors. As more ${ }^{13} \mathrm{C}$ the star have, higher is its ${ }^{7} \mathrm{Li}$ abundance. It seems to confirm, the existence of a single extra mechanism of mixture capable to take both elements from the inner parts to the outer atmosphere.
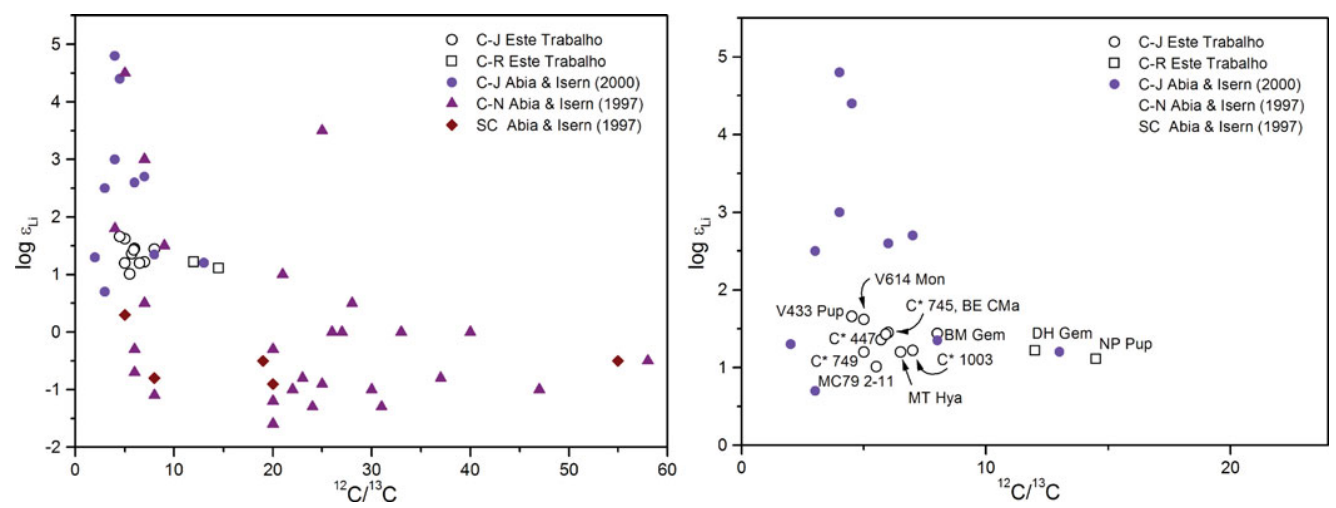

Figure 1. Left panel: Correlation between ${ }^{7} \mathrm{Li}$ and ${ }^{13} \mathrm{C}$ abundance values. Right panel: a close up of the region where our sample is located.

The C-J and their supposed progenitors, C-R, have different areas in the graphic. However, any separation among normal and silicate C-J do not seems to exist, reinforcing the idea that both, as well as the C-R, experiment the same process of production. On the other hand, ${ }^{7} \mathrm{Li}$ and ${ }^{13} \mathrm{C}$ can only be detected if an extra process of mixture takes place (HBB or CBP). Therefore, it is also possible to infer an evolutionary sequence $\mathrm{C}-\mathrm{R} \rightarrow \mathrm{C}-\mathrm{J}$, if we consider that the process capable to enrich with carbon the photosphere also carries ${ }^{7} \mathrm{Li}$ and ${ }^{13} \mathrm{C}$.

\section{References}

Abia et al. 1993, A\&\&A, 275, 96

Abia, C. \& Isern, J. 2000, MNRAS, 177, 89

Evans, T. L. 1990, MNRAS, 243, 336-348

Morris, M. 1987, PASP, 99, 1115-1122

Yamamura et al. 2000, A\&SA, 363, 629-639 